\title{
Infrared light heat therapy of port wine stain in adult patients as new innovative technique
}

\section{Khalifa E. Sharquie', Wesal K. Aljanabi ${ }^{2}$}

\author{
${ }^{1}$ Department of Dermatology, College of Medicine, University of Baghdad, Iraqi and Arab Board for Dermatology \\ and Venereology, Center of Dermatology and Venereology, Medical City, Baghdad, Iraq, ${ }^{2}$ Center of Dermatology and \\ Venereology, Medical City, Baghdad, Iraq
}

Corresponding author: Prof. Khalifa E. Sharquie, E-mail: ksharquie@ymail.com

\begin{abstract}
Background: Port wine needs early age intervention, most effectively by pulse dye laser where about 40-45\% improves only and by at least $75 \%$ after 5 to 25 sessions. Objectives: To try therapy of port wine stain in adult patients using flame of infrared light. Patients and Methods: Twenty adult patients with facial port wine stain were treated during the period from 2014-2017 years, 11 females and 9 males with ages ranged from 16-35 years with a mean of 25 years. After local anesthesia with $2 \%$ xylocaine, direct flame of infrared light that generated from heated red head of needle of diathermy was used. This light therapy was delivered with about $1-2 \mathrm{~cm}$ away from the port wine and the time of exposure was decided by greyish blanching of the lesion, usually 1-2 minutes needed. Only one session of infrared was delivered. The response to therapy and according to the reduction rate was estimated as follow:0-25\%,>26-50\%,>51-75\%,>76-100\%. Results: Immediately after session, there was edema, swelling. Reduction of erythema of port wine started immediately after infrared exposure and was obvious after two weeks and then slowly faded, with more improvement in the next few months. The reduction rate of port wine stain was ranged between $50-70 \%$ after only one session in 19 patients while one patient showed $25 \%$ reduction. It was noticed that, whenever the color of the port wine stain is dusky red with thickened nodular lesions, it responded quickly and better to this therapy. Conclusion: Infrared light heat therapy from diathermy needle was an effective mode of therapy in all adult patients with port wine stain. This new technique is easy to be used and non -costly procedure especially when compared with pulse dye laser.
\end{abstract}

Key words: Port Wine stain; Flame Infra-red; Pulsed Dye laser; Sharquie

\section{INTRODUCTION}

Port-wine stain (PWS) is a congenital slow-flow vascular malformation with worldwide prevalence of 0.3\% [1]. Although PWS have no sex predilection, they are much less common in Asians and African Americans [2]. They occur sporadically alone or as a part of syndrome or inherited as part of associated arteriovenous malformation [3]. It is often unilateral pinkish-red to purple patch that tend to darken and thicken with time, persist lifelong with geographic contour or a dermatomal distribution. Lesions are flat and painless, do not bleed spontaneously, and are never warm on palpation. Acquired port wine stain can be seen after trauma [4]. Fifty percent of cases are located on the face, where they follow the distribution of the trigeminal nerve [3]. The major concern for a patient with PWS is cosmetic because of the visible discoloration. Hypertrophy of soft tissue often like lips and gums and underlying hard tissues can occur with time, especially when it affects the V2 and V3 dermatomes or the extremities. Overgrowth of the maxilla or mandible leads to skeletal asymmetry, occlusal tilt, and open-bite deformity. When the child has atopic dermatitis, psoriasis, or acne, lesions are worse in the area of PWS, a finding known as the 
Meyerson phenomenon [5]. Also evenly thickened skin, purple nodules, and pyogenic granulomas can develop by adolescence and over time [6,7] .

Facial PWS was thought to be caused by clonal expansion of abnormal cells originating from the neural crest. [8] It is proposed that PWS develop due to irregularities in neural development as axonal degeneration and genetic mutations that may be familial or sporadic. Studies have shown that biopsies of PWS specimen have a greater vessel-to-nerve ratio with overall decreased nerve density relative to normal skin. This leads to chronic ischemia and results in further axonal injury and degeneration with worsening of ectatic vessels [2].

Histologically, PWS is characterized by dilated capillaries of the papillary and upper reticular dermis combined with areas of increased number of normallooking capillaries. [9] The endothelial cells are flat. Factor VIII, fibronectin, and basement membrane protein are normal, but $\mathrm{S} 100$ staining shows abnormal innervation $[10,11]$.

The goal of management is to minimize the impact of the PWS on self-esteem and quality of life. Treatment options for PWS have included cosmetic cover-up, skin grafting, radiation, dermabrasion, cryosurgery, tattooing, and electrotherapy [12].

Laser treatment is the gold standard therapy for most PWS. Pulsed-dye laser with its specific wavelength $(585$ or $595 \mathrm{~nm}$ ) and a short pulse duration (400-1500 ms) currently gives the best results in infants and children by lightening the red color. PDL treatment is safe, producing temporary purpura but a low risk of hypo- or hyperpigmentation and scarring. Multiple sessions (6-12) are needed, and general anesthesia may be necessary as the procedure is painful. Laser treatment is more efficient on the cervicofacial and trunk area than on the extremities. Early treatment during childhood does not reduce the number of laser sessions $[13,14]$.

Long-pulse pulsed Alexandrite lasers has been shown to be best for hypertrophic, purple lesions as pulsed dye lasers work best for flat, pink lesions. Also a frequencydoubled (532-nm) Nd: YAG laser that allows for shorter pulse widths, large spot sizes, and high fluencies resulted in up to $75 \%$ improvement in color at 1 month after a single treatment [12].
Infrared (IR) is a type of electromagnetic radiation, including wavelengths between $780 \mathrm{~nm}$ and $1000 \mu \mathrm{m}$. IR is divided into different bands: Near-Infrared

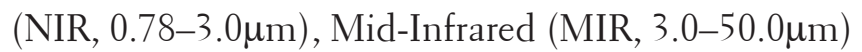

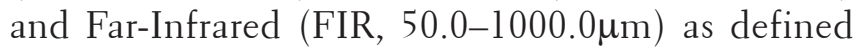
in standard ISO 20473:2007 Optics and photonics Spectral bands [15].

IR radiation can penetrate the epidermis, dermis, and subcutaneous tissue to differing extents depending on the exact wavelength range being studied. Exposure to IR is perceived as heat [16].

Increasing evidence suggests that IR can carry out photo stimulation and photo biomodulation effects particularly benefiting neural stimulation, wound healing, and cancer treatment [16].

The mechanism of IR induced physiological effects is thought to be due to two main types of photo receptors (i.e. cytochrome c oxidase and intracellular water) [17]. Photon absorption converts light into signals that can stimulate biological processes [18]. The action of IR light on water dynamics in membranes, mitochondria and/or cells could modulate signaling pathways, production of reactive oxygen species (ROS), ATP (adenosine triphosphate), $\mathrm{Ca} 2+, \mathrm{NO}$, and inositol phosphates group [19-22]. Secondary effects are always preceded by primary effects, including stress signaling, metabolic processes, cytoskeleton organization, cell proliferation/differentiation, and homeostasis (depending on injury or metabolic redox potentials). [23,24] Additionally, Shapiro et al, 2012 [25] demonstrated that IR light could excite cells through water absorption, with a temperature increases, affecting the plasma membrane and altering the electrical capacitance, thereby depolarizing the target cells [25].

One possible mechanism of Infrared nerve stimulation (INS) is photothermal effects caused by water absorption of energy, rather than photochemical reactions that can occur with radiation possessing greater photon energy (shorter wavelength), or photomechanical pressure waves [26]. The heat sensitive ion channel called "transient receptor potential vanilloid l" (TRPVl) is a possible receptor that is stimulated during INS. TRPVI may be activated thermally by the radiant energy absorbed by water present within the neural tissue [27] .

IR radiation in rats, has shown an increase in the release of the growth factor and anti inflammatory cytokine 
transforming growth factor- $\beta$ l (TGF- $\beta 1$ ) which leads to activation of fibroblasts for improved healing of wounds [28]. Additionally, IR radiation resulted in an increased fibroblast proliferation in vitro [29]. IR can also lead to different expression of type I procollagen, and higher expression of TGF- $\beta 1,-\beta 2$, and $-\beta 3[30,31]$. Also IR radiation causes increase blood vessels probably through up-regulation of vascular endothelial growth factor (VEGF) and the downregulation of the antiangiogenic factor thrombospondin-2 (TSP-2) in the skin epidermis [32].

The beneficial effects of low doses of IR on the skin including photoprotection against UV-induced damage, photo rejuvenation, reduction of pigmented lesions, and fewer fine lines and wrinkles [33].

However, IR-induced free radicals and ROS can be a double-edged sword, at low doses they can activate protective responses, but at high doses ROS can damage organelles and cells in the skin resulting in photoaging [34].

The mechanism of IR radiation damaging the skin is based on upregulation of matrix metalloproteinase-1 (MMP-1). IR also decreases the antioxidant carotenoids in skin [34]. On chronic exposure, Infrared light harmful effects also extend to Tandoor (Tanoor) Poikiloderma, erythema ab igne, skin cancer mainly squamous cell carcinoma and Merkel-cell carcinomas [35]. Furthermore, severe skin aging may develop occasionally on bakers' arms because of exposure to hot ovens and on the faces of glass blowers. In the above examples, the skin was exposed to massive heat via convection through hot air flow, conduction by direct contact and/ or radiation (IR). [30] While intense acute exposure can cause skin burn [36].

Overall, the mechanisms of action of IR radiation can be divided into two broad groups listed below:

1-Energy transfer mechanism: Electrical capacitance of cells regulated by IR Cellular structures (water, proteins, amino acids, lipids, etc.) Exclusion zone generated in water acts as a re-chargeable biological battery interaction between IR and water molecules IR is absorbed and re-radiated as different electromagnetic wavelengths by tissue chromophores [36].

2-Signal pathway mechanism: IR affects cellular redox state in mitochondria and modulates reactive oxygen species and ATP production. Stimulation of nitric oxide, cytochrome oxidase, and transcription factors, cytokines, growth factors, and inflammatory mediators, etc. Signaling via light or heat sensitive ion channels (Ionic pumps and molecular motors). Signaling via cyclic AMP/GMP and G-protein coupled receptors, and inositol phosphate [37]. IR induces in bulk water the release and transport of protons, activating membrane signaling pathways and transmembrane ion channel effects [36,38].

Direct infrared light flame has been effectively used as therapeutic model for treatment of cutaneous Leishmaniasis since long time ago [39-41].

These effective therapeutic trials have encouraged us to conduct the present work using infrared light flame in treatment of port wine stain in adult patients.

\section{PATIENTS AND METHODS}

This is an interventional therapeutic trial where twenty adult patients with port wine stain were treated during the period from 2014-2017, 11 females and 9 males with ages ranged from 16-35 years with a mean of 25 years. Ten patients had thickened nodular lesions on the top of port wine. These patients with PWS were evaluated for demographic features like age, gender and site of lesion. None of the participating patients had received any form of treatment before the present therapy. No associated arteriovenous malformation nor syndromic features were observed.

All lesions were located on the face with exception of one case having lesion on face extending to the neck and ear. The right side was affected in ten patients (five males and five females). Eight patients had lesions on the left side (six females and two male). One female patient had midline glabellar lesion and one male patient with bilateral lesions on cheek. Eleven patients had lesions on V2 (seven males and four females). Seven patients, four females and two males, had V1 trigeminal nerve distribution. One female patient had V1, V2, V3 distribution. One female patient had V2\&V3 distribution. The sizes of the skin lesions in majority of patients were within 0-20 cm2. Direct flame infrared light exposure from heated red electrode of diathermy needle was done after local $2 \%$ xylocaine anesthesia where the source of IFR was $1-2 \mathrm{~cm}$ away from PWS. The time of exposure was until the surface of port wine becomes blanched grayish in color, usually 1-2 minutes were needed. This procedure was repeated until the total 
surface was covered and one session was usually applied. Oral prednisolone $5 \mathrm{mg}$ twice a day, desloratadine tab twice a day together with topical antiseptics and oral antibiotics were given for two weeks and then to be evaluated. Then oral propranolol $40 \mathrm{mg}$ was administered for at least two months to prevent neoangiogenesis. There were five patients with hyperplastic lesions, including nodular and thickened lesions.

Physician Global Assessment [42] was applied to assess the response to therapy which includes "poor improvement" (skin recovery: 0\%-25\%), "moderate improvement" (skin recovery: 26\%-50\%), "significant improvement" (skin recovery: 51\%-75\%), and "cure" (skin recovery: 76\%-100\%). All patients were also screened for any complications.

\section{RESULTS}

Twenty patients with sporadic congenital port wine stain were evaluated for response. There was immediate blanching of redness and the lesion became whitish grayish in color (Fig. 1).

Immediately after session, there was edema, swelling and sometime oozing and then followed by crustation that stayed for two weeks and then fall down leaving pink colour. Reduction of erythema started immediately after infrared exposure and was obvious after two weeks and then slowly more improvement in the next few months. The reduction rate of port wine was ranged between $50 \%-75 \%$ after only one session in $19(95 \%)$ patients while one patient $(5 \%)$ showed $25 \%$ reduction after 2-3 months follow up (Figs. 2 - 5). No relapse in all patients' that were followed up more than two months after therapy. It was noticed that, whenever the color of the port wine stain is dusky red with thickened nodular and plaque lesions, it will respond quickly and better to this therapy while if the port wine stain color is light pink, the response is less and slow. No reported post inflammatory hyperpigmentation or hypopigmentation were observed. One patient developed hypertrophic scar that slowly gone overtime.

\section{DISCUSSION}

In a child, it becomes more challenging to keep the patient stabilized for the duration of the treatment session. In addition, the need for a good level of anesthesia whether general, local or topical with all its medical and psychological impact. All these obstacles and the presence of non-clearing, poorly responding
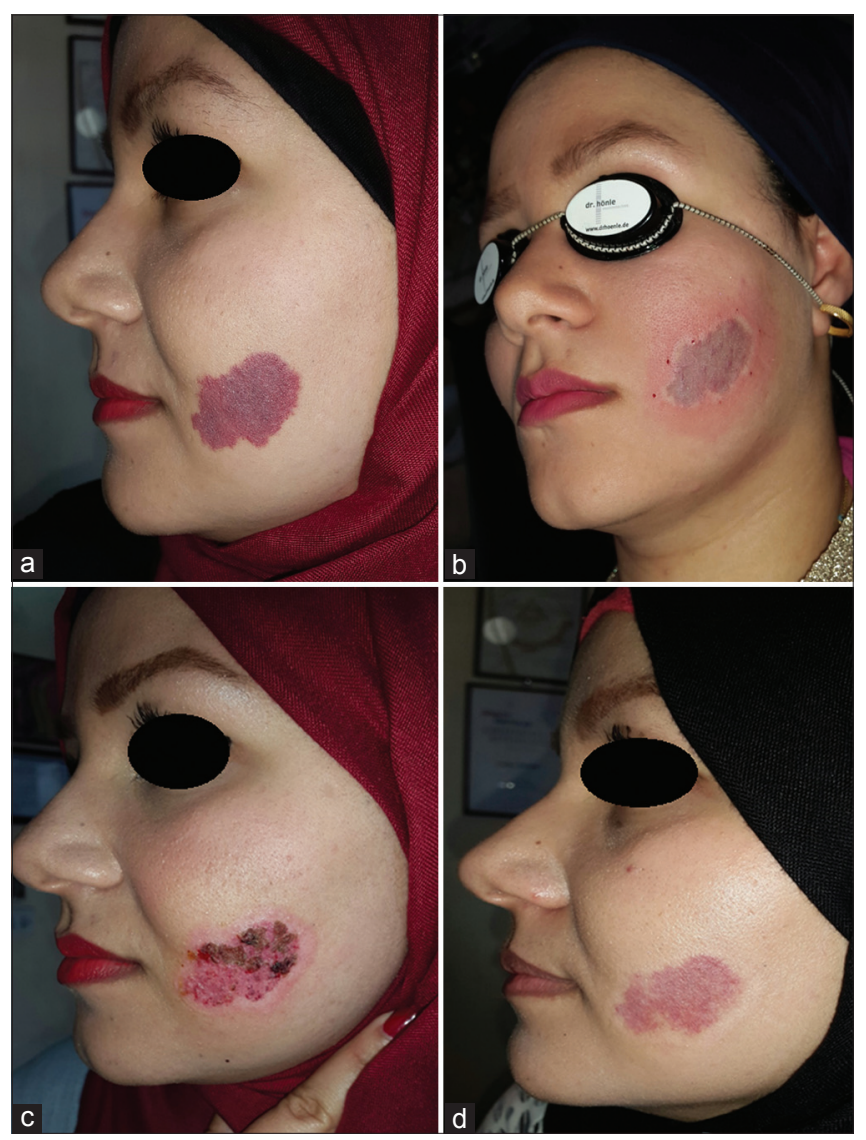

Figure 1: A twenty-two years old female patient with congenital Port Wine Stain treated with one Infrared flame session. (a) before treatment (b) immediate greying and blanching (c) two weeks later (d) after one month.

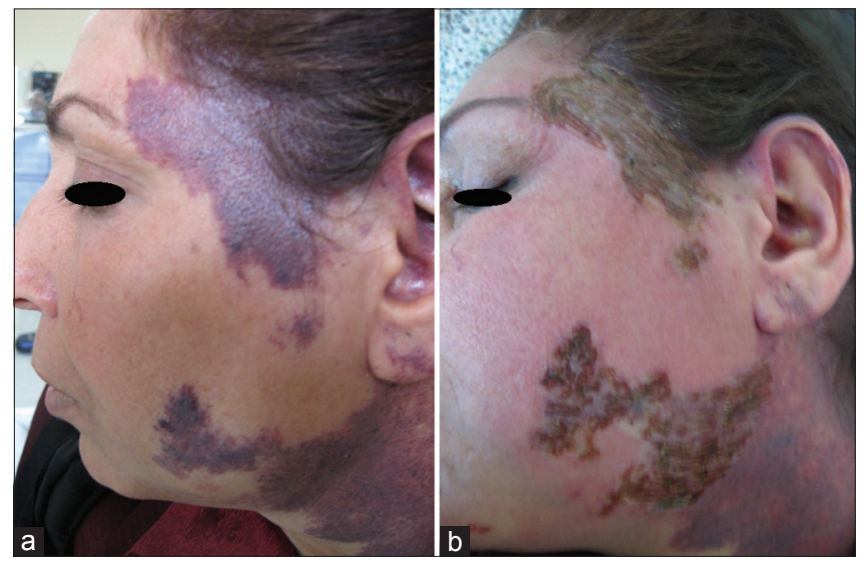

Figure 2: Twenty-nine years old female patient with congenital Port Wine Stain treated with one Infrared light flame session. (a) before treatment with thickened nodular plaques (b) one week after treatment session with crustation.

or resistant lesions to lasers, encourage researchers to make trial of continuously new methods for treatment of port wine stain.

In a review of previous reports on results of treating PWS with PDL, Shi and colleagues reported in their 


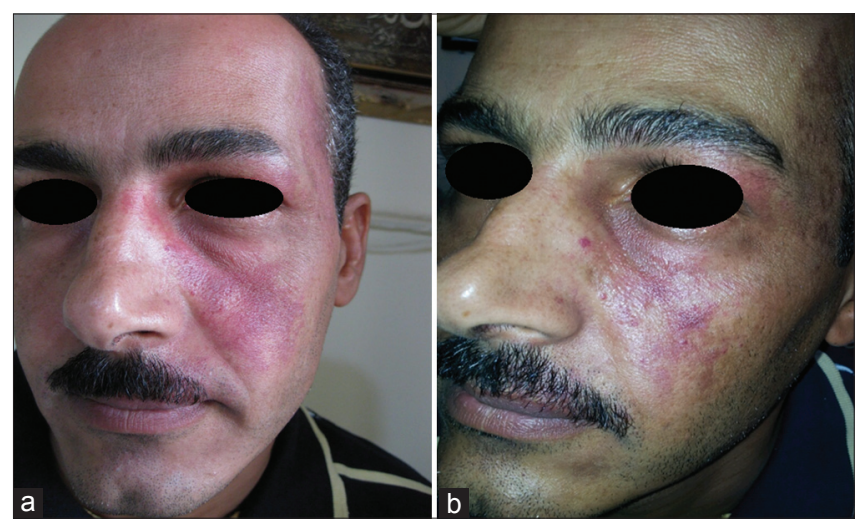

Figure 3: Thirty years old male patient with congenital Port Wine Stain treated with one Infrared flame session. (a) before treatment with thickened lesions (b) two months after one session treatment with significant improvement.

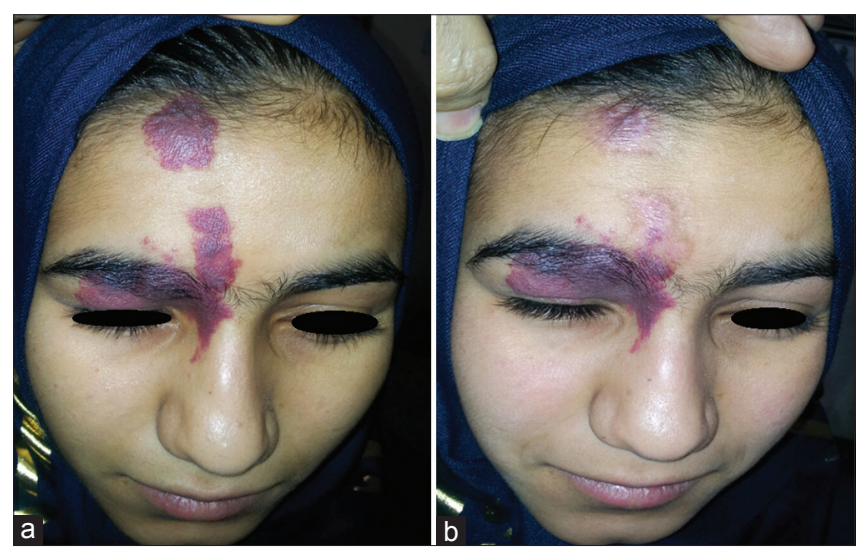

Figure 4: Seventeen years old female patient with congenital Port Wine Stain treated with one Infrared flame session. (a) before treatment with thickened nodular lesions (b) one months after one session treatment with complete clearance of the treated area only.

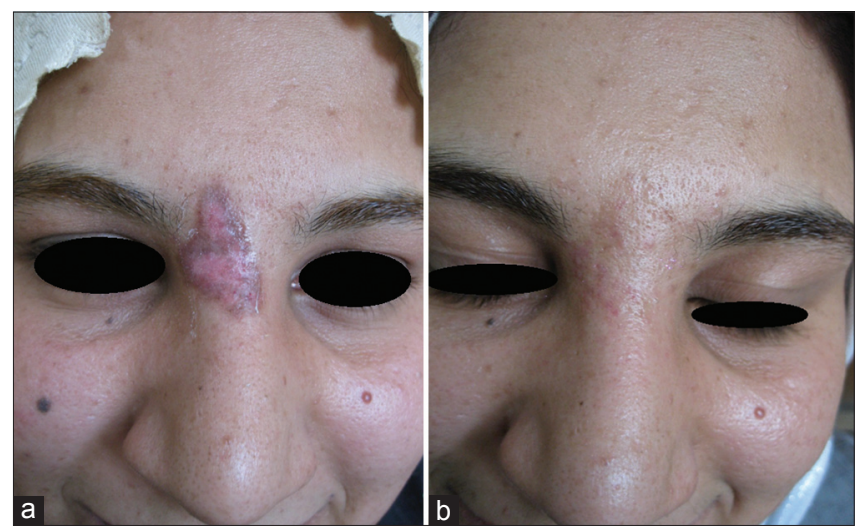

Figure 5: Twenty-five years old female patient with congenital Port Wine Stain treated with one session of Infrared flame. (a) before treatment with thickened plaque (b) two months after therapy.

review of 848 Chinese patients with PWSs where the patients were treated at least three times sessions with $595 \mathrm{~nm}$ PDL (including even a few who were treated about 20 times); The response rate of all PWS treated patients was $69.9 \%$, within which the cure rate was only $6.3 \%$. The response rate to PDL was the highest $(93.9 \%)$ in patients 1 year or younger when compared with older patients, whereas those treated after age 50 reacted the worst $(\mathrm{RR}=25 \%)$. The reactions of the patients with hyperplastic lesion were worse than those with red patches $(\mathrm{RR}=36.4 \%$ vs $71.7 \%)$ [43].

Further study of 197 infants treated with PDL sessions without general nor local anesthesia and a cut off one year the results were: 51 patients $(25.9 \%)$ showed $100 \%$ clearance (mean [range] VAS score of 4.78 [4.5 - 5]); 81 patients $(41.1 \%$ ) showed 76 to $99 \%$ improvement (mean [range] VAS score of 3.91 [3.5 to <4.5]); 44 patients (22.3\%) showed $51 \%$ to $75 \%$ improvement (mean [range] VAS score of 2.86 [2.5 to <3.5]); 13 patients $(6.6 \%)$ showed $26 \%$ to $50 \%$ improvement (mean [range] VAS score of 2.12 [1.5 to <2.5]); and 8 patients (4.1\%) showed 0 to $25 \%$ improvement (mean [range] VAS score of $0.78[0$ to $<1.5]$ ). [44] Hence, in reviewing these two studies, significant level of improvement or recovery $(51-75 \%)$ was scored in $(12.9 \%)$ and $(22.3 \%)$ respectively. $[43,44]$. So, one can conclude that PDL does give cure in high percentage of treated patients in young children while the adult patients with port wine are refractory for this therapy.

Infrared light has been used as effective therapy for many dermatological diseases [16]. While infrared light has been used extensively in treatment of cutaneous Leishmaniasis since long time ago by Prof Sharquie, as Iraq is endemic for this disease, the idea is to raise the lesion temperature to around 42-50 C0 where Leishmania parasite will be killed. The cure rate around $80 \%$ and no complications have been recorded [39,40]. Also infrared light has been used a therapy for patients with Vitiligo with successful encouraging results [45].

These studies have encouraged us to conduct the present work using infrared light flame in treatment of port wine stain. The results of present work showed 5175\% reduction rate of lesions size in 19 (95\%) patients after one session. It was also noticed that whenever the color is dusky red with thickened nodules and plaques will respond quickly and better. To achieve more marked improvement further sessions are essentially needed. No significant side effects except erythema which gradually cleared within two months. The mechanism of action is due to multiple of infrared light effects, all work together to induce occlusion of telangectatic blood vessels inside the lesions. The addition of oral propranolol to this therapy, will probably prevent 
angiogenesis in the treated areas, thus minimizing the chance of relapse.

\section{CONCLUSION}

Infrared flame heat therapy is an effective method of treating of adult congenital PWS and is more cost affordable with less treatment sessions needed to achieve $51-75 \%$ reduction rate improvement especially in patients with thickened port wine. No complications were reported. We encourage light based devices companies to cooperate and to manufacture more sophisticated infrared light machines and develop better techniques. A larger study is recommended to include more patients and younger age group.

\section{Limitation of the study}

This is a preliminary work using one session of therapy; this should be repeated using a high number of infants and adult patients with PWS with multiple repeated sessions one to two months apart.

\section{Abbreviations}

Port Wine Stain: PWS, Pulsed dye laser: PDL, Infrared: IR, Infrared nerve stimulation: INS.

\section{Statement of Human and Animal Rights}

All the procedures followed were in accordance with the ethical standards of the responsible committee on human experimentation (institutional and national) and with the 2008 revision of the Declaration of Helsinki of 1975.

\section{Statement of Informed Consent}

Informed consent for participation in this study was obtained from all patients.

\section{REFERENCES}

1. Jacobs AH, Walton RG. The incidence of birthmarks in the neonate. Pediatrics. 1976;58:218-22.

2. Updyke KM, Khachemoune A. Port-wine stains: a focused review on their management. J Drugs Dermatol. 2017;16:1145-51.

3. Boon LM, Ballieux F, Vikkula M. Vascular Malformations. Kang S, Amagai M, Bruckner A L, Enk A H, Margolis D J, McMchael A J, Orringer J S. In: Fitzpatrick's Dermatology, 9th edition 2019. McGraw-Hill. e Book.2636-46.

4. Adams BB, Lucky AW. Acquired port-wine stains and antecedent trauma: case report and review of the literature. Arch Dermatol. 2000;136:897-9.

5. Hofer T. Meyerson phenomenon within a nevus flammeus. The different eczematous reactions within port-wine stains. Dermatology. 2002;205:180-3.
6. Klapman MH, Yao JF. Thickening and nodules in port-wine stains. J Am Acad Dermatol. 2001;44:300-2.

7. Valeyrie L, Lebrun-Vignes B, Descamps V, Bouscarat F, Grossin $\mathrm{M}$, Bélaïch S, et al. Pyogenic granuloma within port-wine stains: an alarming clinical presentation. Eur J Dermatol. 2002;12:373-5.

8. Etchevers HC, Vincent C, Le Douarin NM, Couly GF. The cephalic neural crest provides pericytes and smooth muscle cells to all blood vessels of the face and forebrain. Development. 2001;128:1059-68.

9. Wassef M, Vanwijck R, Clapuyt P, Boon L, Magalon G. [Vascular tumours and malformations, classification, pathology and imaging]. Ann Chir Plast Esthet. 2006;51:263-81.

10. Finley JL, Clark RA, Colvin RB, Blackman R, Noe J, Rosen S. Immunofluorescent staining with antibodies to factor VIII, fibronectin, and collagenous basement membrane protein in normal human skin and port wine stains. Arch Dermatol. 1982;118:971-5.

11. Breugem CC, Hennekam RC, van Gemert MJ, van der Horst CM. Are capillary malformations neurovenular or purely neural? Plast Reconstr Surg. 2005;115:578-87.

12. Kelly KM, Choi B, McFarlane S, Motosue A, Jung B, Khan MH, et al. Description and analysis of treatments for port-wine stain birthmarks. Arch Facial Plast Surg. 2005;7:287-94.

13. van der Horst CM, Koster PH, de Borgie CA, Bossuyt PM, van Gemert MJ. Effect of the timing of treatment of port-wine stains with the flash-lamp-pumped pulsed-dye laser. N Engl J Med. 1998;338:1028-33.

14. Seukeran DC, Collins P, Sheehan-Dare RA. Adverse reactions following pulsed tunable dye laser treatment of port wine stains in 701 patients. Br J Dermatol. 1997;136:725-9.

15. Vatansever F, Hamblin MR. Far infrared radiation (FIR): its biological effects and medical applications. Photonics Lasers Med. 2001;2:4-6.

16. Tsai S-R, Hamblin MR. Biological effects and medical applications of infrared radiation. J Photochem Photobiol B: Biology. 2017;170:197-207.

17. Passarella S, Karu T. Absorption of monochromatic and narrow band radiation in the visible and near IR by both mitochondrial and non-mitochondrial photoacceptors results in photobiomodulation. J Photochem Photobiol B. 2014;140:344-58.

18. Bashkatov AN, Genina EA, Kochubey VI, Tuchin VV. Optical properties of human skin, subcutaneous and mucous tissues in the wavelength range from 400 to $2000 \mathrm{~nm}$. J Phys D: Applied Physics. 2005;38:2543-55.

19. Karu TI. Mitochondrial signaling in mammalian cells activated by red and near-IR radiation. Photochem Photobiol. 2008;84:1091-9.

20. de Freitas LF, Hamblin MR. Proposed Mechanisms of Photobiomodulation or Low-Level Light Therapy. IEEE J Sel Top Quantum Electron. 2016;22:2561201.

21. Irvine RF, Schell MJ. Back in the water: the return of the inositol phosphates. Nat Rev Mol Cell Biol. 2001;2:327-38.

22. Santana-Blank LA, Rodríguez-Santana E, Santana-Rodríguez KE. Photo-infrared pulsed bio-modulation (PIPBM): a novel mechanism for the enhancement of physiologically reparative responses. Photomed Laser Surg. 2005;23:416-24.

23. Calles C, Schneider M, Macaluso F, Benesova T, Krutmann J, Schroeder P. Infrared A Radiation influences the skin fibroblast transcriptome: mechanisms and consequences. J Invest Dermatol. 2010;130:1524-36.

24. Rojas JC, Gonzalez-Lima F. Neurological and psychological applications of transcranial lasers and LEDs. Biochem Pharmacol. 2013;86:447-57.

25. Shapiro MG, Homma K, Villarreal S, Richter C-P, Bezanilla F. Infrared light excites cells by changing their electrical capacitance. Nature Communications. 2012;3:736.

26. Wells J, Kao C, Konrad P, Milner T, Kim J, Mahadevan-Jansen A, et al. Biophysical mechanisms of transient optical stimulation of peripheral nerve. Biophys J. 2007;93:2567-80. 
27. Suh E, Izzo Matic A, Otting M, Walsh J, Richter C-P. Optical stimulation in mice lacking the TRPV1 channel: SPIE; 2009.

28. Toyokawa H, Matsui Y, Uhara J, Tsuchiya H, Teshima S, Nakanishi $\mathrm{H}$, et al. Promotive effects of far-infrared ray on full-thickness skin wound healing in rats. Exp Biol Med. 2003;228:724-9.

29. Vinck EM, Cagnie BJ, Cornelissen MJ, Declercq HA, Cambier DC. Increased fibroblast proliferation induced by light emitting diode and low power laser irradiation. Lasers Med Sci. 2003;18:95-9.

30. Cho S, Shin MH, Kim YK, Seo JE, Lee YM, Park CH, et al. Effects of infrared radiation and heat on human skin aging in vivo. J Investig Dermatol Symp Proc. 2009;14:15-9.

31. Kim MS, Kim YK, Cho KH, Chung JH. Regulation of type I procollagen and MMP-1 expression after single or repeated exposure to infrared radiation in human skin. Mech Ageing Dev. 2006;127:875-82.

32. Kim MS, Kim YK, Cho KH, Chung JH. Infrared exposure induces an angiogenic switch in human skin that is partially mediated by heat. Br J Dermatol. 2006;155:1131-8.

33. Huang Y-Y, Chen ACH, Carroll JD, Hamblin MR. Biphasic dose response in low level light therapy. Dose Response. 2009;7:358-83.

34. Darvin ME, Gersonde I, Albrecht H, Zastrow L, Sterry W, Lademann J. In vivo Raman spectroscopic analysis of the influence of IR radiation on the carotenoid antioxidant substances betacarotene and lycopene in the human skin. Formation of free radicals. Laser Physics Letters. 2007;4:318-21.

35. Miller K, Hunt R, Chu J, Meehan S, Stein J. Erythema ab igne. Dermatol Online J. 2011;17:28.

36. Barolet D, Christiaens F, Hamblin MR. Infrared and skin: Friend or foe. J Photochem Photobiol B. 2016;155:78-85.

37. Santana-Blank L, Rodríguez-Santana E, Santana-Rodríguez K. Theoretic, Experimental, Clinical Bases of the Water Oscillator Hypothesis in Near-Infrared Photobiomodulation. Photomedicine and Laser Surgery. 2010;28:S-41-S-52.

38. Rohani M, Pollack GH. Flow through horizontal tubes submerged in water in the absence of a pressure gradient: mechanistic considerations. Langmuir. 2013;29:6556-61.

39. Jabbar A, Junaid N. Treatment of cutaneous leishmaniasis with infrared heat. Int J Dermatol. 1986;25:470-2.

40. Sharquie KE, Al-Rubaie AW. Infra-red therapy of cutaneous leishmaniasis (Baghdad Boil) Diploma Disseretation, Deparment of Dermatology, College of Medicine, University of Baghdad. 1990.

41. Sharquie KE, Al-Mashhadani SA, Noaimi AA, Al-Zoubaidi WB. Microwave Thermotherapy: New treatment for cutaneous leishmaniasis. Our Dermatol Online. 2015;6:125-9.

42. Currie CL, Monk BE. Can the response of port-wine stains to laser treatment be reliably assessed using subjective methods? $\mathrm{Br} J$ Dermatol. 2000;143:360-4.

43. Shi W, Wang J, Lin Y, Geng J, Wang H, Gong Y, et al. Treatment of port wine stains with pulsed dye laser: a retrospective study of 848 cases in Shandong Province, People's Republic of China. Drug Des Devel Ther. 2014;8:2531-8.

44. Jeon H, Bernstein LJ, Belkin DA, Ghalili S, Geronemus RG. Pulsed Dye Laser Treatment of Port-Wine Stains in Infancy Without the Need for General Anesthesia. JAMA Dermatol. 2019;155:435-41.

45. Sharquie KE, Amin WA. Infrared therapy of localized vitiligo. Board thesis submitted to Dermatology and venereology Scientific Committee, Iraqi Board for Medical Specialization 2007.

Copyright by Khalifa E. Sharquie, et al. This is an open access article distributed under the terms of the Creative Commons Attribution License, which permits unrestricted use, distribution, and reproduction in any medium, provided the original author and source are credited. Source of Support: Nil, Conflict of Interest: None declared. 